Copyright (C) 2013 IEEE. Personal use of this material is permitted. Permission from IEEE must be obtained for all other uses, in any current or future media, including reprinting/republishing this material for advertising or promotional purposes, creating new collective works, for resale or redistribution to servers or lists, or reuse of any copyrighted component of this work in other works. 


\title{
Design of Robust Broadband Beamformers with Discrete Coefficients and Least Squared Criterion
}

\author{
Hai Huyen Dam and Sven Nordholm, Senior Member, IEEE \\ Curtin University of Technology \\ Perth, Westerns Australia
}

\begin{abstract}
This paper investigates the performance of robust and non-robust broadband beamformers with least squares and discrete coefficients to achieve low complexity and efficient hardware implementation. The broadband beamformer coefficients are expressed as the sum of power-of-two terms with a restriction on the total number of power-of-two terms for the beamformer coefficients. An iterative algorithm is employed to reduce the number of non-zero coefficients and thereby multiplier in both the robust and non-robust beamformers. A quantization scheme in combination with a random search is then applied to efficiently distribute the power-of-two terms for the beamformer coefficients. Design examples show that the number of non-zero coefficients for the beamformers can be significantly reduced without a significant degradation in the integral squared error. In addition, robust beamformers are shown to be less sensitive to non-zero coefficient reduction and quantization than non-robust beamformers.
\end{abstract}

\section{INTRODUCTION}

In various speech communication applications, such as hands-free mobile telephony and hearing aids, the recorded microphone signals are corrupted by interference such as background noise and reverberation. The interference causes speech signal degradation, which can lead to errors in speech recognition and speech coding systems [1]-[5]. The goal is to achieve efficient signal enhancement algorithms by removing additive noise without distorting the underlying signal.

Broadband beamformers have been extensively studied due to their wide application in many areas such as radar, sonar, imaging, wireless communications, speech and acoustics [1] [8]. The two well-known multi-microphone speech enhancement techniques are: fixed and adaptive beamformers. In [6], [7] interpolation schemes are developed for image processing with coefficients expressed as the sum of signed power-oftwo (SOSPT) terms. The impact of performance degradation from an ideal system in VLSI circuits and DSP has been investigated. In [8], a multi-stage uniform coefficient (MSUC) filter is proposed. The proposed structure is compared with the previously-proposed signed-power-of-two FIR (SPOT-FIR) filters. This approach, however, is more suitable for image

Hai Huyen Dam is with Department of Mathematics and Statistics, Curtin University of Technology, Perth, Australia

Sven Nordholm is with Department of Electrical and Computer Engineering, Curtin University of Technology, Perth, Australia

Email $\{$ H.Dam,S.Nordholm $\} @$ curtin.edu.au

This research was supported by ARC Discovery Project DP120103859.

Copyright (c) 2013 IEEE. Personal use of this material is permitted. However, permission to use this material for any other purposes must be obtained from the IEEE by sending an email to pubs-permissions@ieee.org processing as the solution can deviate significantly from the desired response, especially at the cut-off frequencies.

In this paper, we concentrate on the design of fixed beamforming [4]-[5]. It is well known that fixed broadband beamformers using small-size microphone arrays are highly sensitive to errors in the microphone characteristics. Thus, robust beamformer design algorithms have been developed to reduce the sensitivity of the beamformers by incorporating the array characteristics model into the algorithm.

Here, we investigate the performance of low complexity non-robust and robust broadband beamformers under least squares with coefficients expressed as the sum of SOSPT terms [10]-[12]. These beamformers are attractive for hardware implementation as multiplications can be efficiently reduced to simple operations of shifts and adds. Since the number of beamformer coefficients is typically large, an iterative algorithm is employed to reduce the number of non-zero coefficients for the non-robust and robust beamformers [13]. For a fixed number of non-zero beamformer coefficients, an efficient quantization procedure combined with a random search algorithm is employed to search for the SOSPT coefficients around the infinite precision solution. Design examples show that the number of non-zero coefficients for the beamformers can be reduced significantly without a significant increase in the integral squared error. In addition, the robust beamformer is less sensitive to non-zero coefficient reduction than the non-robust beamformer. Also, the robust beamformer with SOSPOT coefficients can achieve approximately the same integral squared error as the infinite precision solution with the total number of power-of-two terms approximately twice the total number of beamformer coefficients.

\section{ARRAY RESPONSE}

Consider the design of a microphone array with $N$ elements. For simplicity, we consider far-field signaling modeling and a linear microphone array. Here, each microphone is connected to an $L$-dimensional FIR filter with real coefficients, $\mathbf{h}_{n}=$ $\left[h_{n}(0), \ldots, h_{n}(L-1)\right]^{T}, 0 \leq n \leq N-1$. The response of the broadband beamformer at a normalized frequency $\omega \in \Omega$ and an angle $\phi \in \Phi$ is given by

$$
G(\omega, \phi)=\sum_{n=0}^{N-1} \sum_{\ell=0}^{L-1} h_{n}(\ell) e^{-j \ell \omega} A_{n}(\omega, \phi)
$$

where $A_{n}(\omega, \phi)=e^{-j \omega f_{s} d_{n} \cos \phi / c}$ is the array response at the $n^{\text {th }}$ microphone, $f_{s}$ is the sampling rate, $c$ is the speed of 
sound and $d_{n}$ is the distance between the $n^{\text {th }}$ microphone and the center of the microphone array. Denote by $\mathbf{h}$ an $N L \times 1$ coefficient vector, $\mathbf{h}=\left[\mathbf{h}_{0}, \ldots, \mathbf{h}_{N-1}\right]^{T}$, then the beamformer response in (1) can be expressed as

$$
G(\omega, \phi)=\mathbf{h}^{T} \mathbf{g}(\omega, \phi)
$$

where $\mathbf{g}(\omega, \phi)$ is an $N L \times 1$ vector,

$$
\begin{aligned}
\mathbf{g}(\omega, \phi)= & {\left[e^{-j \omega f_{s} d_{0} \cos \phi / c}, \ldots, e^{-j \omega f_{s} d_{N-1} \cos \phi / c}\right]^{T} } \\
& \otimes\left[1, \ldots, e^{-j(L-1) \omega}\right]^{T}
\end{aligned}
$$

and $\otimes$ represents the Kronecker product.

\section{RobUST AND NON-ROBUST BEAMFORMER DESIGN}

Denote by $H_{d}(\omega, \phi)$ the desired beamformer response at a frequency $\omega$ and an angle $\phi$. The design of non-robust beamformer with least squared criterion can be formulated as minimizing the integral squared error [4]

$$
\begin{aligned}
J(\mathbf{h}) & =\int_{\Omega} \int_{\Phi}\left|H(\omega, \phi)-H_{d}(\omega, \phi)\right|^{2} d \phi d \omega \\
& =\mathbf{h}^{T} \int_{\Omega} \int_{\Phi} \mathbf{Q}(\omega, \phi) d \phi d \omega \mathbf{h}-2 \mathbf{h}^{T} \int_{\Omega} \int_{\Phi} \mathcal{R}(\mathbf{p}(\omega, \phi)) d \phi d \omega \\
& +\int_{\Omega} \int_{\Phi}\left|H_{d}(\omega, \phi)\right|^{2} d \phi d \omega
\end{aligned}
$$

where $\mathbf{Q}(\omega, \phi)=\mathcal{R}\left(\mathbf{g}(\omega, \phi) \mathbf{g}^{H}(\omega, \phi)\right), \quad \mathbf{p}(\omega, p)=$ $\mathbf{g}(\omega, \phi) H_{d}^{*}(\omega, \phi)$ and $\mathcal{R}(\cdot)$ denotes the real part. Thus,

$$
J(\mathbf{h})=\mathbf{h}^{T} \mathbf{Q h}-2 \mathbf{h}^{T} \mathcal{R}(\mathbf{p})+c
$$

where $\mathbf{Q}=\int_{\Omega} \int_{\Phi} \mathbf{Q}(\omega, \phi) d \phi d \omega, \quad \mathbf{p}=\int_{\Omega} \int_{\Phi} \mathbf{p}(\omega, \phi) d \phi d \omega$ and $c$ is a constant. The least squared solution is given by $\mathbf{h}_{L S}=\mathbf{Q}^{-1} \mathcal{R}(\mathbf{p})$.

It is well-known that the least square solution is highly sensitive to errors in microphone characteristics such as gain, phase and microphone position, especially for small size microphone arrays [5]. Thus, the design of a robust beamformer with microphone characteristics must be taken into account [5]. Here, we assume that each microphone has a variation of the form $c e^{-j \gamma}$ where $c$ and $\gamma$ are independent random variations for the amplitude and the phase, respectively. As such, the robust squared error deviation $J_{r}(\mathbf{h})$ is obtained by integrating the squared error deviation over the possible regions of the microphone characteristics. To simplify the model, we assume that all microphones characteristics have the same probability density functions (pdf), $f_{\alpha}(c)$ and $f_{\mathcal{G}}(\gamma)$, for their magnitude and phase, respectively. In general, the model for the pdf can be obtained from the microphone manufacturers.

As with [5], the integral squared error for the robust beamformer can be given as

$$
J_{r}(\mathbf{h})=\mathbf{h}^{T} \mathbf{Q}_{r} \mathbf{h}-2 \mathbf{h}^{T} \mathbf{p}_{r}+c_{r}
$$

where $\mathbf{Q}_{r}=\left(\left(\mu_{c}^{2} \sigma_{\gamma}^{c} \mathbf{1}_{N}+\left(\sigma_{c}^{2}-\mu_{c}^{2} \sigma_{\gamma}^{c}\right) \mathbf{I}_{N}\right) \otimes \mathbf{1}_{L}\right) \odot \mathbf{Q}$ and $\mathbf{p}_{r}=\mu_{c}\left(\mu_{\gamma}^{c} \mathcal{R}(\mathbf{p})+\mu_{\gamma}^{\mathbf{s}} \mathcal{I}(\mathbf{p})\right)$. Here, $\mu_{c}=\int_{c} c f_{\alpha}(c) d c, \sigma_{c}^{2}=$ $\int_{c} c^{2} f_{\alpha}(c) d c$ and $\mu_{\gamma}^{c}=\int_{\gamma} \cos (\gamma) f_{\mathcal{G}}(\gamma) d \gamma$ and $\mu_{\gamma}^{s}=$ $\int_{\gamma} \sin (\gamma) f_{\mathcal{G}}(\gamma) d \gamma$. Also $\sigma_{\gamma}^{c}=\left(\mu_{\gamma}^{c}\right)^{2}+\left(\mu_{\gamma}^{s}\right)^{2}$ where $\mathbf{1}_{N}$ represents an $N \times N$ matrix with all elements being one and $\mathbf{I}_{N}$ is an $N \times N$ identity matrix. In addition, $\odot$ represents matrix element-to-element multiplication, $\mathcal{I}(\cdot)$ denotes the imaginary part of a complex number and $c_{r}$ is a constant. The robust least square solution is given by $\mathbf{h}_{r, L S}=\left(\mathbf{Q}_{r}\right)^{-1} \mathbf{p}_{r}$.

\section{Reduced COMPlexity Non-Robust And Robust BEAMFORMERS}

As the number $N L$ of coefficients for the beamformer is large, an iterative algorithm developed in [13] is employed to reduce the number of non-zero coefficients for both the nonrobust and robust least squares beamformers. For each iteration, the number of non-zero coefficients in the beamformer is reduced by one by setting one coefficient to zero. The process is repeated until the least squared error reaches a certain limit. A summary of the procedure is given as follows:

Procedure 1: Reduce the number of non-zero coefficients given a requirement $e$ for the integral squared error deviation.

- Step 0: Set $\tilde{\mathbf{h}}$ as the least squares solution.

- Step 1: Reduce the number of non-zero coefficient in the beamformer by one by setting one non-zero coefficient to zero. This non-zero coefficient is chosen so that is has the least magnitude. Update the least squares solution $\tilde{\mathbf{h}}$.

- Step 1: Calculate the corresponding integral squared error. If the error is less than $e$, then go to Step 1. Otherwise, update $\tilde{\mathbf{h}}$ as the last solution with the error less than $e$. Insert zeros at the correct positions in $\tilde{\mathbf{h}}$ and output the solution with a reduced number of non-zero coefficients.

In the following section, the design of a non-robust/robust beamformer with SOSPT terms is investigated to reduce operations associated with the beamformers to shifts and adds.

\section{NON-RobUST AND ROBUST BEAMFORMERS WITH SOSPT COEFFICIENTS}

The non-zero coefficients in the beamformer is expressed in terms of SOSPT terms with a maximum number of $B$ bits for each term. We denote the quantized vector for $\tilde{\mathbf{h}}$ with $K$ non-zeros terms as $\tilde{\mathbf{h}}_{q}$.

Denote by $c_{k}$, the number of power-of-two terms in the coefficient $\tilde{h}_{q}(k)$, where $c_{k} \leq B$ and $0 \leq k \leq N L-1$. Then, the coefficient $\tilde{h}_{q}(k)$ can be expressed as $\tilde{h}_{q}(k)=\sum_{k=1}^{c_{k}} \eta_{k} 2^{-\nu_{k}}$ where $\eta_{k}$ is a binary value, $\eta_{k} \in\{-1,1\}$ and $\nu_{k}$ is an integer in the set $\{1, \ldots, B\}$. Thus, the range for each SOSPT is $\left[-1+2^{-B}, 1-2^{-B}\right]$. The total number power-of-two terms for the beamformer is restricted to a positive number $M$ according to

$$
\sum_{k=0}^{N L-1} c_{k} \leq M
$$

We extend the quantization method [12] to the beamformer coefficients with the infinite precision solution $\tilde{\mathbf{h}}$. The quantization procedure is summarized as follows.

Procedure 2 Quantization procedure for $\tilde{\mathbf{h}}$ given an upper bound $M$ on the total number of power-of-two terms.

- Step 1: Initialize $\tilde{\mathbf{h}}_{q}$ as a zero vector and set $k=1$. 
- Step 2: Search for an index $\ell, 0 \leq \ell \leq N L-1$, corresponding to the coefficient in $\tilde{\mathbf{h}}$ with the maximum absolute value, $|\tilde{h}(n)|=\max _{0 \leq \ell \leq N L-1}|\tilde{h}(\ell)|$. We have the following two cases: If $|\tilde{h}(n)|<2^{-B-1}$, then stop the procedure. Otherwise, search for a power-of-two term that is closest to $\tilde{h}(n)$,

$$
\left|\tilde{h}(n)-\zeta_{1}\right|=\min _{\zeta \in \mathcal{B}}|\tilde{h}(n)-\zeta|
$$

where $\mathcal{B}=\left\{-2^{-B}, \ldots,-2^{-1}, 2^{-1}, \ldots, 2^{-B}\right\}$. Locate the $k^{\text {th }}$ power-of-two term to the $n^{\text {th }}$ position of $\tilde{\mathbf{h}}_{q}$. Update the quantized vector $\tilde{\mathbf{h}}_{q}$ by adding $\zeta_{1}$ to the coefficient $\tilde{h}_{q}(n)$ and update $\tilde{\mathbf{h}}$ by subtracting $\zeta_{1}$ from $\tilde{h}(n)$. If $k<M$, then set $k:=k+1$ and return to the beginning of Step 2. Otherwise, stop the procedure. The vector $\tilde{\mathbf{h}}_{q}$ is the quantized solution with a restriction of $M$ on the total number of power-of-two terms.

Since each beamformer coefficient is not restricted to a fixed number of power-of-two terms, there is a degree of freedom in distributing the power-of-two terms to the appropriate beamformer coefficients. Here, a random search is employed to generate random candidate SOSPOT coefficients in the neighbourhood of $\tilde{\mathbf{h}}$ so as to search for the optimal discrete solution. More precisely, the quantization Procedure 2 is employed over a random coefficient vector $\tilde{\mathbf{h}}+\alpha \tilde{\mathbf{h}}_{r}$ generated by adding a random vector $\tilde{\mathbf{h}}_{r}$ with non-zero coefficients in similar positions as $\tilde{\mathbf{h}}$ and elements in the range $[-1,1]$ to the original vector $\tilde{\mathbf{h}}$. The value $\alpha$ is a scale factor that controls the size of neighbourhood to be searched. The random search is performed for a number of iterations and the quantized solution with minimum integral squared error is saved. Note that the random search algorithm is similar to the mutation of genetic algorithm and the random walk in simulated annealing [10], [14].

\section{Design EXAMPLES}

Consider the design of an equispaced linear broadband beamformer with $N=20$ and the common spacing of $0.04 \mathrm{~m}$ between microphone elements. The speed of sound is $c=343 \mathrm{~ms}^{-1}$. The desired beamformer has the spectral passband $[200,3800] \mathrm{Hz}$ and the spectral stopband $[0,100] \cup$ $[3950,4000] \mathrm{Hz}$ with a sampling rate of $8000 \mathrm{~Hz}$. Also, the spatial passband is $\left[0,15^{\circ}\right]$ and the spatial stopband is $\left[25^{\circ}, 180^{\circ}\right]$.

The beamformer desired response $H_{d}(\omega, \phi)$ is given by

$$
H_{d}(\omega, \phi)= \begin{cases}e^{-j \omega N_{d}}, & (\omega, \phi) \in \Omega_{p} \times \Phi_{p} \\ 0, & \text { otherwise }\end{cases}
$$

where $\Omega_{p}, \Phi_{p}$ denote the spectral and spatial passbands, respectively, and $N_{d}$ is the desired delay. Here, the magnitude and the phase of the microphone array are modelled as uniform distributions in the intervals $[0.995,0.105]$ and $[-0.005,0.005]$, respectively. For the random search, chosen values for $\alpha$ and the number of iterations are $\alpha=0.001$ and 500 , respectively.

Figs. 1 and 2 plot the integral squared errors for the robust and non-robust beamformers with $L=40$ and $L=60$, respectively. The beamformers are designed with $N_{d}=20$. For $L=40$, the number of non-zero beamformer coefficients increases from 231 (robust) and 284 (non-robust) to 800 . Similarly, for $L=60$, the number of non-zero coefficients increases from 230 (robust) and 296 (non-robust) to 1200. In all cases, Procedure 1 stops when the integral squared error reaches $-14 \mathrm{~dB}$. It can be seen from the plots that the number of non-zero coefficients can be reduced significantly with a small increase in the integral squared error. In fact, for the robust beamformers, the number of non-zero coefficients can be reduced by $50 \%$ with only a $0.3-0.6 \mathrm{~dB}$ increase in the integral squared error. In addition, the robust beamformer is less sensitive to non-zero coefficient reduction than the non-robust beamformer. In particular, the two beamformers achieve approximately the same performance with 371 nonzero coefficients.

Figs. 3 and 4 show the maximum absolute value of the robust and non-robust beamformer coefficients for $L=40$ and $L=60$ when the number of non-zero coefficients $K$ increases from 800 and 1200, respectively. In both cases, the non-robust beamformers have significantly larger magnitude coefficients than the robust beamformers. As such they are more sensitive to non-zero coefficients reduction and quantization than the robust beamformers.

Fig. 5 shows the beampattern for the non-robust beamformer with $L=40$ and 800 coefficients. Fig. 6 shows the beampatterns for the robust beamformers: (i) with 800 coefficients and (ii) the number of non-zero coefficients reduced to 231 . The beampattern for robust beamformer with a reduced number of coefficients is only slightly higher than that of the robust beamformers with full length.

Table I shows the integral squared error $[\mathrm{dB}]$ for the robust and non-robust beamformers with $L=40$ and $L=60$, respectively. In [15], the mean of the squared beam pattern with the probability density functions of the microphone errors taken into account is used to measure the performance of the perturbed beamformer. Here, we employ a Monte Carlo simulation with 500 variations in the magnitude and the phase of the microphone characteristics. The last column in the Table I shows the error for the non-robust least square beamformer degrades significantly when there are variations in the microphone characteristics, while the error of the robust beamformer reduces only slightly.

Fig. 7 plots the integral squared errors for the optimized robust beamformers with SOSPT coefficients for $L=40$. The total number of power-of-two terms for the beamformer coefficients increases from 400 to 1800 . The figure shows the discrete beamformer solution starting from different infinite precision solutions: (i) robust beamformer with 287 non-zero coefficients and integral squared error of $-14.5 \mathrm{~dB}$; (ii) robust beamformer with 371 non-zero coefficients and $-15.0 \mathrm{~dB}$; (ii) robust beamformer with 493 non-zero coefficients and $-15.5 \mathrm{~dB}$; and (iv) robust beamformer with 607 non-zero coefficients and $-15.8 \mathrm{~dB}$. The figure also shows the optimal solution for a fixed total number of power-of-two terms. It can be seen that the discrete beamformer requires at most twice the total number of beamformer coefficients to reach approximately the same integral squared error as the robust 
infinite precision solution.

Fig. 8 shows the integral squared errors for the optimized robust beamformers with SOSPT coefficients and $L=60$. As with the previous plot, the figure shows: (i) robust beamformer with 230 non-zero coefficients and $-14.5 \mathrm{~dB}$; (ii) robust beamformer with 272 non-zero coefficients and $-15.0 \mathrm{~dB}$; (ii) robust beamformer with 332 non-zero coefficients and $-15.5 \mathrm{~dB}$; and (iv) robust beamformer with 523 non-zero coefficients and $-15.8 \mathrm{~dB}$. Observe that for the same total number of power-of-two terms, the integral squared error for the beamfomer with $L=60$ is only slightly lower than that with $L=40$. In addition, the integral squared error for the discrete solution with approximately 1400 total power-of-two is close to the infinite precision solution.

\section{CONCLUSIONS}

This paper investigates the performance of non-robust and robust least squares beamformers with a reduced number of non-zero coefficients and SOSPOT terms to reduce the computational complexity associated with the beamformers. Design examples show that the robust beamformer is less sensitive than the non-robust beamformer with respect to coefficient reduction and quantization. In addition, the number of non-zero coefficients can be significantly reduced without a significant reduction in the total integral squared error.

\section{REFERENCES}

[1] L. C. Parra, "Steerable Frequency-invariant Beamforming for Arbitrary Arrays," J. Acoust. Soc. Amer., vol. 119, pp. 3839-3847, 2006.

[2] G. W. Elko, "Microphone Array Systems for Hands-free Telecommunicaition," Speech Commun., vol. 20, no. 1-3, pp. 229-240, Dec. 1996.

[3] E. Mabande, A. Schad, and W. Kellermann, "Design of Robust Superdirective Beamformers as a Convex Optimization Problem," IEEE Int. Conf. Acoust. Speech, Signal Processing, pp. 77-80, Apr. 2009.

[4] C. C. Lai, S. Nordholm, and Y.-H. Leung, "Design of Robust Steerable Broadband Beamformers Incorporating Microphone Gain and Phase Error Characteristics," IEEE International Conference Acoustics, Speech and Signal Processing (ICASSP), pp. 101-104, May 2011.

[5] S. Doclo and M. Moonen, "Design of Broadband Beamformers Robust Against Gain and Phase Errors in the Microphone Array Characteristics," IEEE Trans. Signal Processing, vol. 51, no. 10, pp. 2511-2526, Oct. 2003.

[6] M. Karaman, A. Atalar, H. Koymen, "VLSI circuits for adaptive digital beamforming in ultrasound imaging," IEEE Trans. Med. Imaging, vol. 12, pp. 711-720, 1993.

[7] C. R. Hazard and G. R. Lockwood, "Theoretical assessment of a synthetic aperture beamformer for real-time 3-D imaging," IEEE Trans. Ultrason. Ferroelectr. Freq. Control, vol. 46, pp. 972-980, 1999.

[8] F. K. Schneider, Y.M. Yoo, A. Agarwal, L.M. Koh and Y. Kim, "New demodulation filter in digital phase rotation beamforming", Ultrasonics, vol. 44, pp. 265-271, 2006.

[9] V. V. Zaharov and M. Teixeira, "SMI-MVDR Beamformer Implementations for Large Antenna Array and Small Sample Size," IEEE Trans. Circuits Syst. I, vol. 55, no. 10, pp. 3317-3327, 2008

[10] C. K. S. Pun, Y. C. Wu, S. C. Chan, and K. L. Ho, "On the Design and Efficient Implementation of the Farrow Structure," IEEE Signal Processing Letters, vol. 10, no. 7, pp. 189-192, Jul. 2003.

[11] H. H. Dam, A. Cantoni, K. L. Teo and S. Nordholm, "FIR Variable Digital Filter With Signed Power-of-Two Coefficients," IEEE Trans. Circuits Syst. I, vol. 54, no. 6, pp. 1348-1357, Jun. 2007.

[12] Y. C. Lim, "Design of Discrete-Coefficient-Value Linear Phase FIR Filters with Optimum Normalized Peak Ripple Magnitude," IEEE Trans. Circuits Systems, vol. 37, no. 12, pp. 1480-1486, Dec. 1990.

[13] D. Rimantho, H. Dam, and S. Nordholm, "Design of Low Complexity Robust Broadband Beamformers with Least Squared Performance Criterion," Advances in Information Technology and Applied Computing, vol. 1, pp. 46-50, Nov. 2012.
[14] D. Karaboga and D. T. Pham, Intelligent Optimization Techniques: Genetic Algorithms, Tabu Search, Simulated Annealing and Neural Networks. Berlin, Germany: Springer-Verlag, 2000.

[15] M. Crocco and A. Trucco, "Stochastic and Analytic Optimization of Sparse Aperiodic Arrays and Broadband Beamformers with Robust Superdirective Patterns," IEEE Trans. Audio, Speech and Language Processing, vol. 20, no. 9, pp. 2433-2447, Nov. 2012.

\begin{tabular}{|c|c|c|c|c|c|}
\hline$L$ & Coeff. & $\begin{array}{c}\text { Non-zero } \\
\text { coefficients }\end{array}$ & & $\begin{array}{c}\text { Without } \\
\text { variation }\end{array}$ & $\begin{array}{c}\text { With } \\
\text { variation }\end{array}$ \\
\hline \multirow{4}{*}{40} & \multirow{2}{*}{$\begin{array}{c}\text { Full } \\
\text { length }\end{array}$} & $K=800$ & Non-robust & $-17.96 \mathrm{~dB}$ & $33.54 \mathrm{~dB}$ \\
\cline { 4 - 6 } & $\begin{array}{c}\text { Reduced } \\
\text { length }\end{array}$ & $K=284$ & Robust & $-15.94 \mathrm{~dB}$ & $-15.55 \mathrm{~dB}$ \\
\cline { 3 - 6 } & & $K=231$ & Robust & $-14.01 \mathrm{~dB}$ & $35.85 \mathrm{~dB}$ \\
\hline 60 & $\begin{array}{c}\text { Full } \\
\text { length }\end{array}$ & $K=1200$ & Non-robust & $-19.57 \mathrm{~dB}$ & $-14.80 \mathrm{~dB}$ \\
\cline { 3 - 6 } & $\begin{array}{c}\text { Reduced } \\
\text { length }\end{array}$ & $K=296$ & Robust & $-16.29 \mathrm{~dB}$ & $-15.84 \mathrm{~dB}$ \\
\cline { 3 - 6 } & & Non-robust & $-14.00 \mathrm{~dB}$ & $32.08 \mathrm{~dB}$ \\
\hline
\end{tabular}

TABLE I

INTEGRAL SQUARE ERRORS FOR ROBUST AND NON-ROBUST BEAMFORMERS WITH FULL LENGTH OR A REDUCED NUMBER OF NON-ZERO COEFFICIENTS. THE COEFFICIENTS HAVE INFINITE PRECISION.

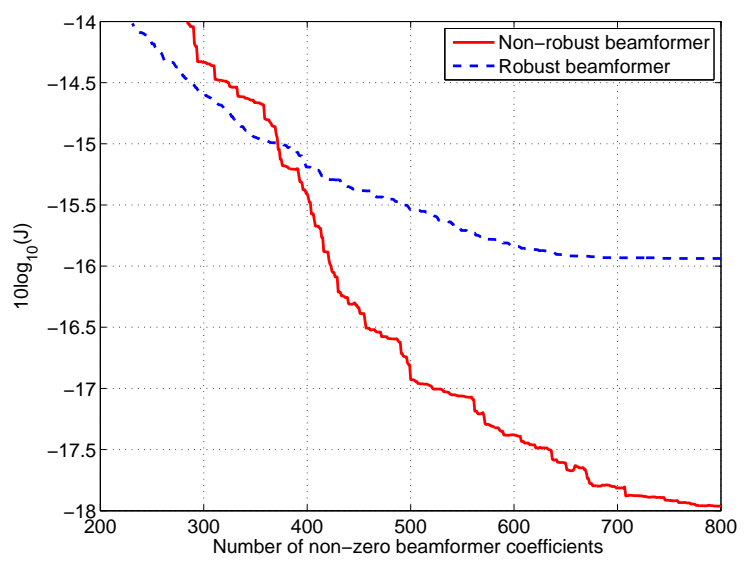

Fig. 1. Robust and non-robust beamformers with $L=40$ when the number of non-zero coefficients increases from 284 (non-robust) or from 231 (robust) to 800 .

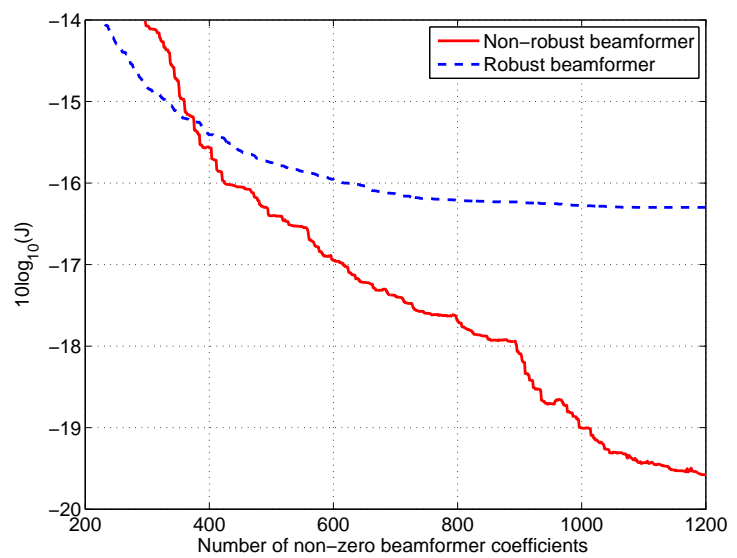

Fig. 2. Robust and non-robust beamformers with $L=60$ when the number of non-zero coefficients increases from 296 (non-robust) or from 230 (robust) to 1200 . 

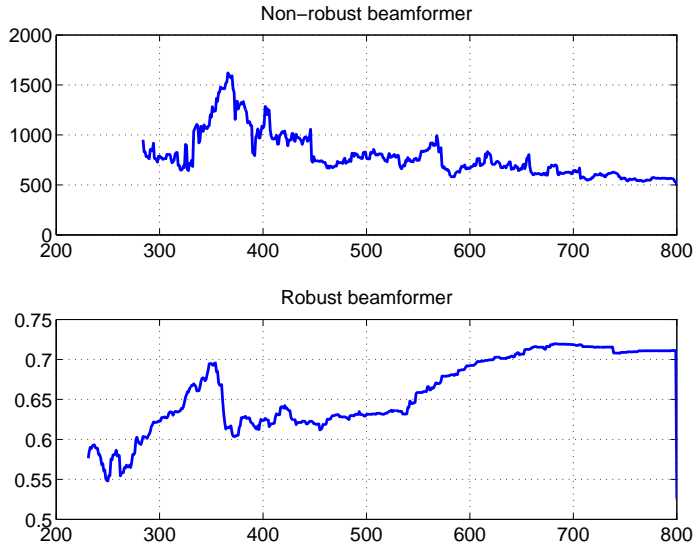

Fig. 3. Maximum magnitude coefficients. The number of non-zero coefficients increases from 284 (non-robust) and 231 (robust) to $800(L=40)$.
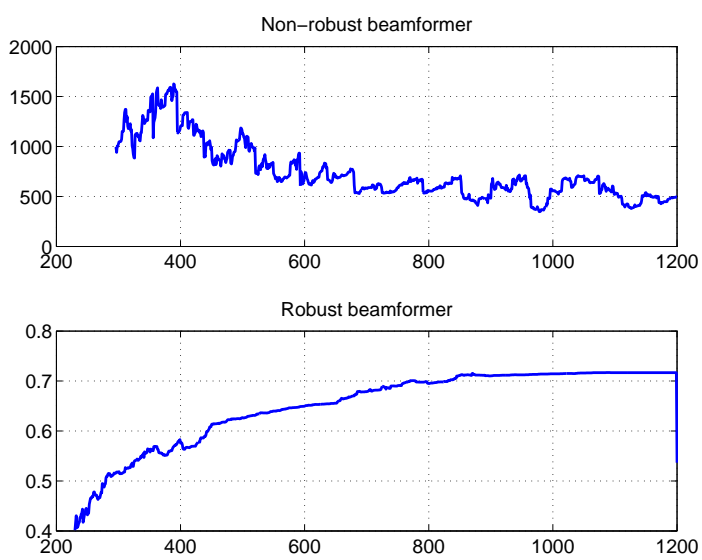

Fig. 4. Maximum magnitude coefficients. The number of non-zero coefficients increases from 296 (non-robust) and 230 (robust) to $1200(L=60)$.

Non-robust beamformer with full length

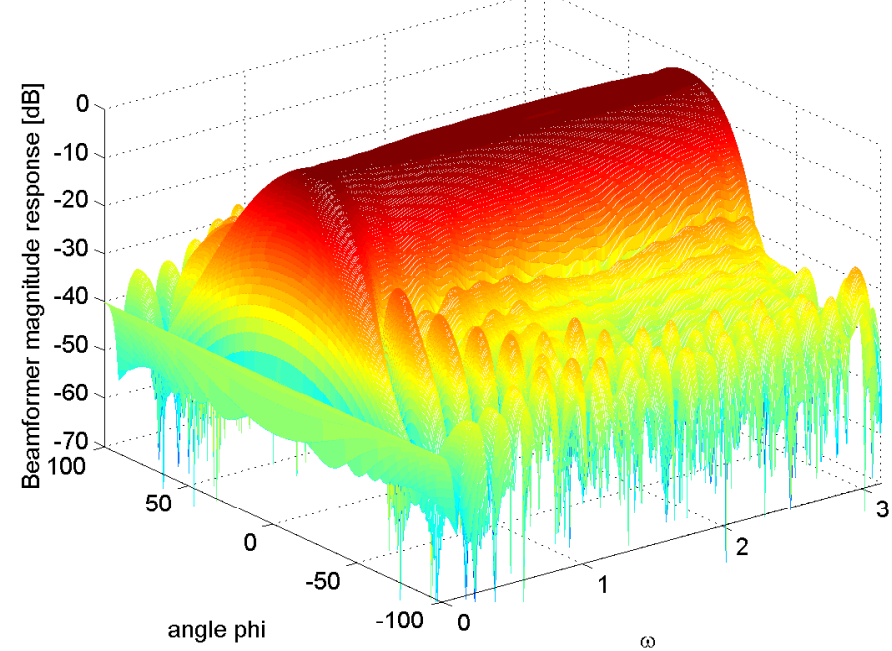

Fig. 5. Magnitude response for non-robust beamformer with $L=40$.

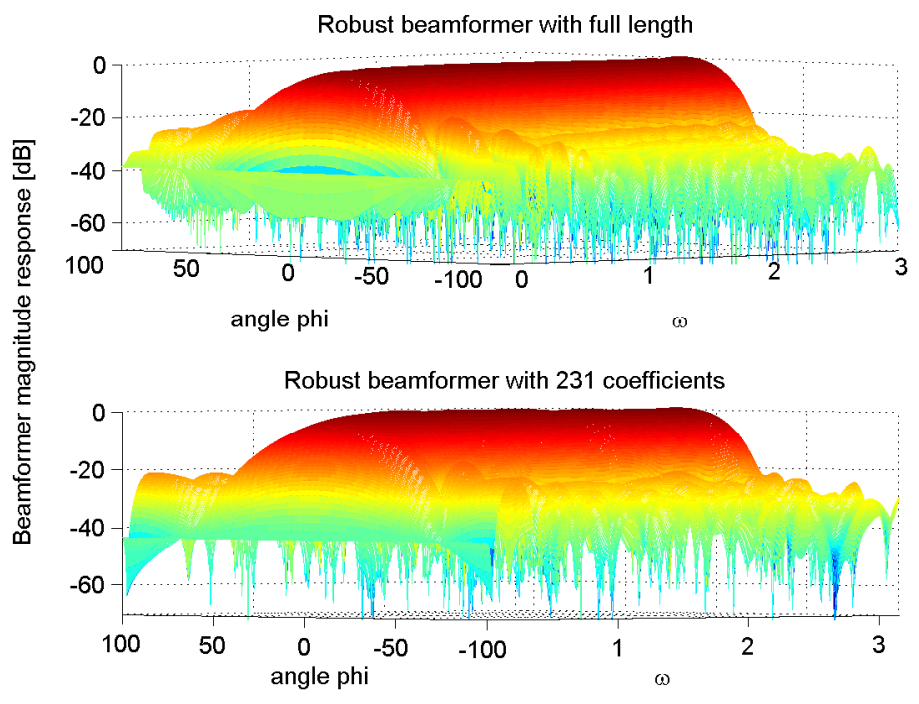

Fig. 6. Magnitude response for robust beamformer with $L=40$.

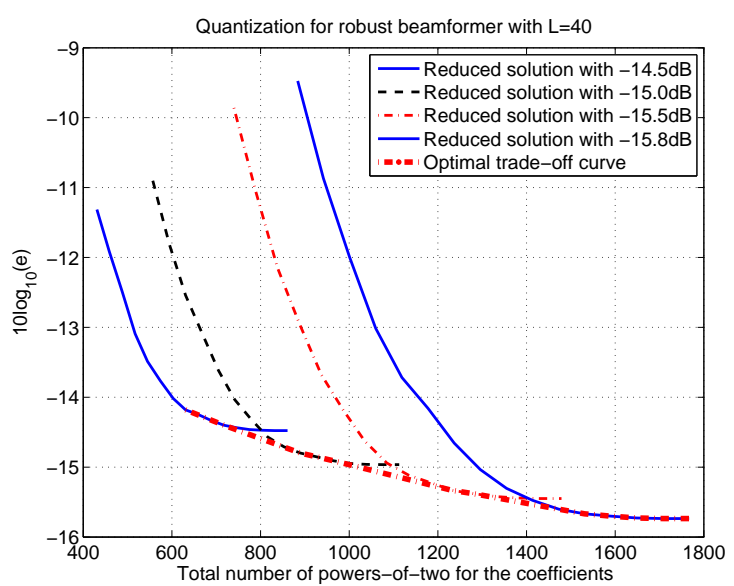

Fig. 7. Robust beamformer with different numbers of non-zero coefficients and different restrictions on the total number of power-of-two terms.

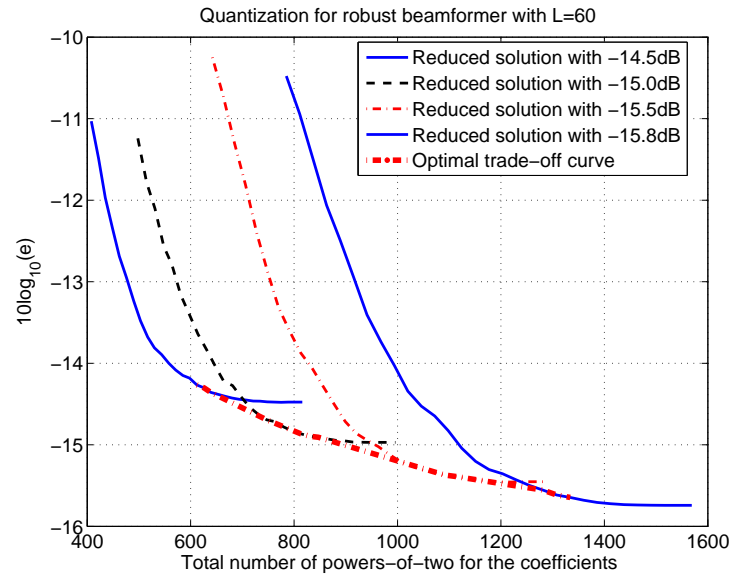

Fig. 8. Robust beamformer with different numbers of non-zero coefficients and different restrictions on the total number of power-of-two terms. 